\title{
Air Quality Monitoring System Using Wireless Sensor Networks
}

\author{
Mrs. S. Mahalakshmi M.E \\ Lecturer (Senior Grade), Department of Electronics \\ and Communication Engineering, VSVN Polytechnic \\ College, Virudhunagar, Tamil Nadu, India
}

\author{
N. Ambika M.E \\ Research Scholar, Department of Electronics and \\ Communication Engineering, SSN College of \\ Engineering, Chennai, Tamil Nadu, India
}

\section{ABSTRACT}

In real-time accurate and sensitive data collection under varying temperature and humidity microenvironments. Additionally, because the velocities of the particles change under varying temperature and humidity conditions according to the Ideal Gas Law, it is important for the system to be examined separately under each variable condition in a controlled manner. For instance, the water particles produced under changing humidity can interfere with the dust particle measurements in the optical sensor, and as a result, increase the sampled particle concentration. Another inaccurate reading can be caused when the air temperature increases, as the velocity of the dust particles that pass across the optical sensor increase. Consequently, the wireless sensor was calibrated by observing the results of the professional Dylos and the wireless sensor separately under these varying conditions. Then, we conducted two different tests to verify the functionality and durability of the sensor. These tests were performed for 24 hours both indoors and outdoors to test the reliability and accuracy of the wireless sensors against the Dylos air quality monitoring. In this system, we proposed wireless sensor network to monitor the temperature, humidity and also the gas leakage of industrial applications .By introducing this we can monitor the sensor parameter values in wireless communication and also we can upload the sensor parameter values in web portal. For Wireless communication we are using here is WSN, which helps to collect all nodes data's and can be monitored in PC and can also upload in web portal so that for anywhere in this world we can monitor these conditions.

KEY WORDS: Wireless sensor network, Air quality Monitoring system (AQMS), Gas sensor (NO2, CO)

\section{INTRODUCTION}

Air plays a very important part in our life, we will talk about just a few of them today! Air contains oxygen, which is essential for life on Earth. We inhaled air, taking the out oxygen and other gases our bodies need, then air we exhaled is made up of the gases useless to us - mainly carbon-dioxide. Moving air, called wind, has great force. It enables the movement of sailboats and gliders. It runs windmills, which are used to generate electricity. Wind also helps in the dispersal of seeds.

Water, air, and soil are three natural resources that we cannot live without. The Forest Service strives to protect, maintain, and restore these valuable assets now and into the future. Water is one of the most important natural resources flowing from forests. The organisms living in the soil respire through oxygen present in this air. Hence plants and animals are interdependent and they maintain a balance between carbon dioxide and oxygen in the atmosphere. The article briefly describes the importance of air for survival. Moving air (wind) is also important for some plants to pollinate. Animals including humans need oxygen to live. We breathe in oxygen and breathe out Carbon Dioxide. There are also air pockets in soils and water that help tiny living things survive in water and beneath the soils.

The human body uses air in several ways. Without it we wouldn't be able to live. Breathing in Oxygen - Air supplies the oxygen to our lungs which circulates through our bodies to combine with sugars in the food we eat to give us energy and to build tissues. Exhaling Waste - Oxygen is primarily combined with carbon in our bodies to produce Carbon Dioxide which is exhaled. Other trace gases are exhaled including a little methane and water vapor. The water vapor is not really a waste product like $\mathrm{CO} 2$ or methane. 
Regulating Temperature - The air around us helps to keep us warm. In the absence of any heat in the air, our bodies lose heat quickly. If we are working hard or exercising we generate excess heat. The air provides some cooling to help the body maintain an ideal temperature of $37^{\circ} \mathrm{C}$.

\section{PROBLEM DESCRIPTION}

Air quality affects your health. Ozone and particulate matter, also known as PM, are two pollutants of significant concern in the Bay Area. Ozone is primarily a health problem in the summertime, and $\mathrm{PM}$ in the winter. Poor air quality affects your health. When pollution is bad, it can irritate your eyes, nose, and throat, cause shortness of breath, aggravate asthma and other respiratory conditions, and even affect your heart and cardiovascular system. Breathing polluted air for long periods of time can cause more serious health problems. Two primary causes of poor air quality in the Bay Area are ozone and particulate matter, also called PM or soot. Cars and trucks and other mobile sources are the major sources of ozone in the summertime. In the winter, wood smoke from woodstoves and fireplaces is the major source of PM pollution.

\section{PROJECT DESCRIPTION}

Recently the air is get polluted. Due to the polluted air Many disease will occur like asthma etc. Hence we propose the Air quality monitored system based on the arduino controller and different sensors. Based on the sensed value of the sensor If there is each value is high. We can upload the sensor parameter values in web portal using IOT technology.

\section{LITERATURE SURVEY}

\section{A review on the human health impact of airborne particulate matter.}

Particulate matter (PM) is a key indicator of air pollution brought into the air by a variety of natural and human activities. As it can be suspended over long time and travel over long distances in the atmosphere, it can cause a wide range of diseases that lead to a significant reduction of human life. The size of particles has been directly linked to their potential for causing health problems. Small particles of concern include "inhalable coarse particles" with a diameter of 2.5 to $10 \mu \mathrm{m}$ and "fine particles" smaller than $2.5 \mu \mathrm{m}$ in diameter As the source-effect relationship of PM remains unclear, it is not easy to define such effects from individual sources such as long-range transport of pollution. Because of the potent role of PM and its associated pollutants, detailed knowledge of their human health impacts is of primary importance. This paper summarizes the basic evidence on the health effects of particulate matter. An in-depth analysis is provided to address the implications for policy-makers so that more stringent strategies can be implemented to reduce air pollution and its health effects.

\section{Health effects of fine particulate air pollution: lines that connect.}

Efforts to understand and mitigate the health effects of particulate matter (PM) air pollution have a rich and interesting history. This review focuses on six substantial lines of research that have been pursued since 1997 that have helped elucidate our understanding about the effects of PM on human health. There has been substantial progress in the evaluation of PM health effects at different timescales of exposure and in the exploration of the shape of the concentration-response function. There has also been emerging evidence of PM-related cardiovascular health effects and growing knowledge regarding interconnected general pathophysiological pathways that link PM exposure with cardiopulmonary morbidity and mortality. Despite important gaps in scientific knowledge and continued reasons for some skepticism, a comprehensive evaluation of the research findings provides persuasive evidence that exposure to fine particulate air pollution has adverse effects on cardiopulmonary health. Although much of this research has been motivated by environmental public health policy, these results have important scientific, medical, and public health implications that are broader than debates over legally mandated air quality standards.

\section{Climate change, air pollution and extreme} events leading to increasing prevalence of allergic respiratory diseases.

The prevalence of asthma and allergic diseases has increased dramatically during the past few decades not only in industrialized countries. Urban air pollution from motor vehicles has been indicated as one of the major risk factors responsible for this increase. Although genetic factors are important in the development of asthma and allergic diseases, the rising trend can be explained only in changes occurred in the environment. Despite some differences in the air pollution profile and decreasing trends of some key air pollutants, air quality is an important concern for public health in the cities throughout the world. 
Due to climate change, air pollution patterns are changing in several urbanized areas of the world, with a significant effect on respiratory health. The observational evidence indicates that recent regional changes in climate, particularly temperature increases, have already affected a diverse set of physical and biological systems in many parts of the world. Associations between thunderstorms and asthma morbidity in pollinosis subjects have been also identified in multiple locations around the world. Allergens patterns are also changing in response to climate change and air pollution can modify the allergenic potential of pollens especially in presence of specific weather conditions. The underlying mechanisms of all these interactions are not well known yet. The consequences on health vary from decreases in lung function to allergic diseases, new onset of diseases, and exacerbation of chronic respiratory diseases. Factor clouding the issue is that laboratory evaluations do not reflect what happens during natural exposition, when atmospheric pollution mixtures in polluted cities are inhaled. In addition, it is important to recall that an individual's response to pollution exposure depends on the source and components of air pollution, as well as meteorological conditions. Indeed, some air pollution-related incidents with asthma aggravation do not depend only on the increased production of air pollution, but rather on atmospheric factors that favour the accumulation of air pollutants at ground level. Considering these aspects governments worldwide and international organizations such as the World Health Organization and the European Union are facing a growing problem of the respiratory effects induced by gaseous and particulate pollutants arising from motor vehicle emissions.

4. On the use of small and cheaper sensors and devices for indicative citizen-based monitoring of respirable particulate matter

Respirable particulate matter present in outdoor and indoor environments is a health hazard. The particle concentrations can quickly change, with steep gradients on short temporal and spatial scales, and their chemical composition and physical properties vary considerably. Existing networks of aerosol particle measurements consist of limited number of monitoring stations, and mostly aim at assessment of compliance with air quality legislation regulating mass of particles of varying sizes. These networks can now be supplemented using small portable devices with low-cost sensors for assessment of particle mass that may provide higher temporal and spatial resolution if we understand the capabilities and characteristics of the data they provide. This paper overviews typical currently available devices and their characteristics. In addition it is presented original results of measurement and modelling in the aim of one low-cost PM monitor validation.

\section{HIPAA compliant wireless sensing smartwatch application for the self-management of pediatric asthma}

Asthma is the most prevalent chronic disease among pediatrics, as it is the leading cause of student absenteeism and hospitalization for those under the age of 15 . To address the significant need to manage this disease in children, the authors present a mobile health (mHealth) system that determines the risk of an asthma attack through physiological and environmental wireless sensors and representational state transfer application program interfaces (RESTful APIs). The data is sent from wireless sensors to a smart watch application (app) via a Health Insurance Portability and Accountability Act (HIPAA) compliant cryptography framework, which then sends data to a cloud for real-time analytics. The asthma risk is then sent to the smart watch and provided to the user via simple graphics for easy interpretation by children. After testing the safety and feasibility of the system in an adult with moderate asthma prior to testing in children, it was found that the analytics model is able to determine the overall asthma risk (high, medium, or low risk) with an accuracy of $80.10 \pm 14.13 \%$. Furthermore, the features most important for assessing the risk of an asthma attack were multifaceted, highlighting the importance of continuously monitoring different wireless sensors and REST ful APIs. Future testing this asthma attack risk prediction system in pediatric asthma individuals may lead to an effective self-management asthma program.

\section{Efficiency Improvement of an Integrated Giant Freshwater-White Prawn Farming in Thailand Using a Wireless Sensor Network.}

Most local freshwater prawn farmers in Thailand are currently relying their prawn production on a traditional farming approach, which requires highlyexperienced farm manager, effort and time. By continuing with this inefficient approach, it is hard to improve the productivity. It possible to use in monitoring and control of greenhouse parameter in precision agriculture 


\section{PROPOSED SYSTEM}

In the existing system wireless sensor network to monitor the temperature, humidity and also the gas leakage of industrial applications .Our propose the air quality monitored system based on the Arduino microcontroller with different Sensors and IOT module board. Based on the sensed value of the sensor, if there is each value is high then the alert message will be send to the corresponding mobile using Global System for Mobile communication. By introducing this we can monitor the sensor parameter values in wireless communication and also we can upload the sensor parameter values in web portal. For Wireless communication we are using here is WSN, which helps to collect all nodes data's and can be monitored in PC and can also upload in web portal so that for anywhere in this world we can monitor these conditions.

\section{SYSTEM DESIGN}

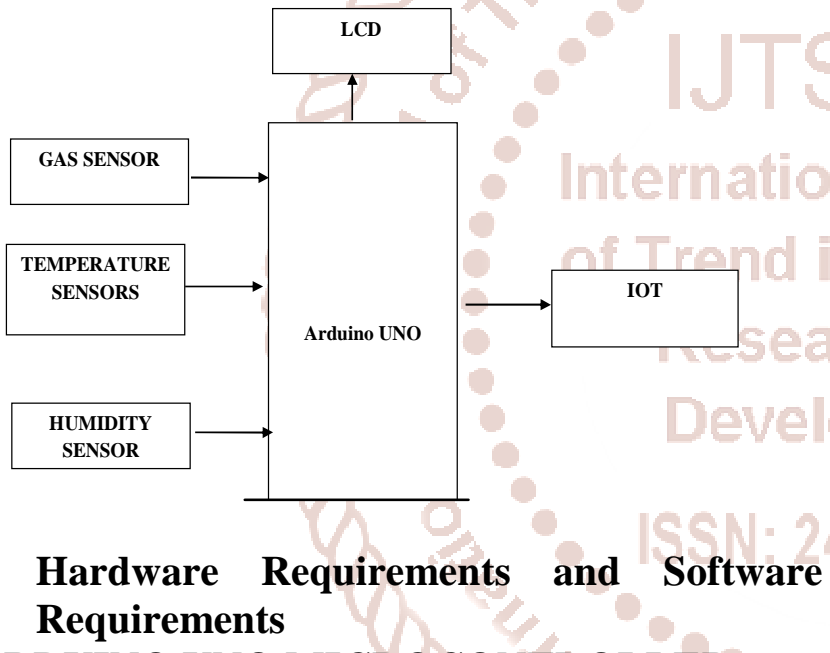

\section{ARDUINO UNO MICROCONTROLLER}

Arduino is an open-source electronics platform based on easy-to-use hardware and software. Arduino boards are able to read inputs - light on a sensor, a finger on a button, or a Twitter message - and turn it into an output - activating a motor, turning on an LED, publishing something online. You can tell your board what to do by sending a set of instructions to the microcontroller on the board. To do so you use the Arduino programming language (based on Wiring), and the Arduino Software (IDE), based on Processing.

\section{IOT MODULE}

The Internet of Things (IoT) is a system of interrelated computing devices, mechanical and digital machines, objects, animals or people that are provided with unique identifiers and the ability to transfer data over a network without requiring humanto-human or human-to-computer interaction.

\section{LCD DISPLAY}

A liquid-crystal display (LCD) is a flat-panel display or other electronically modulated optical device that uses the light-modulating properties of liquid crystals. Liquid crystals do not emit light directly, instead using a backlight or reflector to produce images in colour or monochrome.

\section{GAS SENSOR}

A gas detector is a device that detects the presence of gases in an area, often as part of a safety system. This type of equipment is used to detect a gas leak or other emissions and can interface with a control system so a process can be automatically shut down.

\section{TEMPERATURE SENSOR}

A temperature sensor is a device, typically, a thermocouple or RTD, that provides for temperature measurement through an electrical signal. A thermocouple (T/C) is made from two dissimilar metals that generate electrical voltage in direct proportion to changes in temperature.

\section{ARDUINO SKETCH IDE}

The Arduino Integrated Development Environment or Arduino Software (IDE) - contains a text editor for writing code, a message area, a text console, a toolbar with buttons for common functions and a series of menus. It connects to the Arduino and Genuino hardware to upload programs and communicate with them.

\section{EMBEDDED C}

Embedded $\mathrm{C}$ is a set of language extensions for the C programming language by the $\mathbf{C}$ Standards Committee to address commonality issues that exist between $\mathbf{C}$ extensions for different embedded systems.

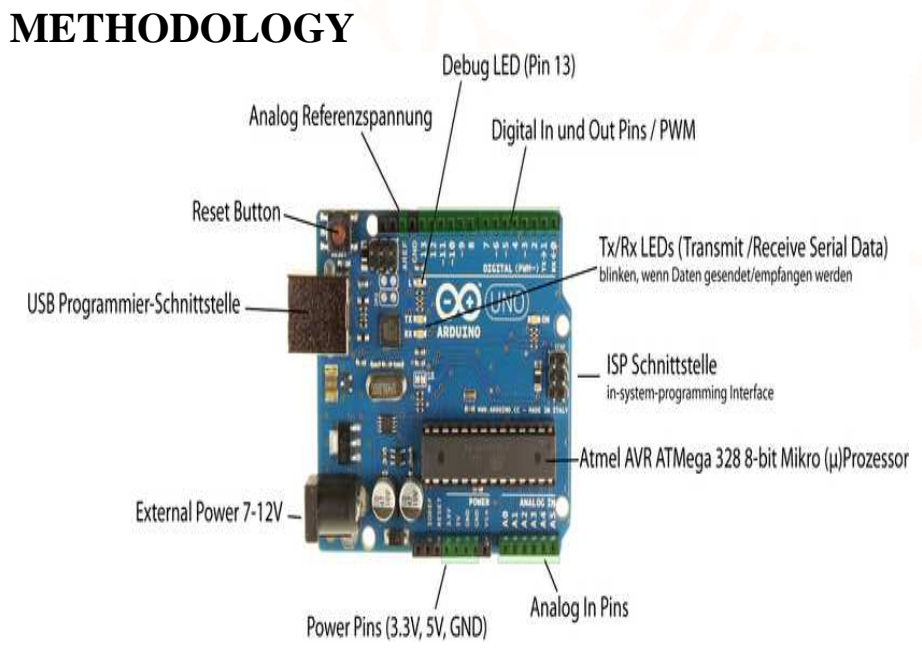


In our process, arduino used to collect the information from the 14- digital input/output pin's and Analog PORT pins. Compiling the code before upload. If this is your first time you've ever compiled code to your Arduino before plugging it in to the computer go to the Tools menu, then Serial Port and take note of what appears there. Plug your Arduino UNO board in to the USB cable and into your computer. In our process, arduino used to collect the information form the 14digital input/output pin's and Analog PORT pin's. Compiling the code before upload. If this is your first time you've ever compiled code to your Arduino before plugging it in to the computer go to the Tools menu, then Serial Port and take note of what appears there. Plug your Arduino UNO board in to the USB cable and into your computer.

\section{Analog to Digital Conversion}

Arduino has six different Analog to digital conversion pin. This pin's to connect the six different Analog output product sensor. They are named as (A0, A1, A2, A3, A4, A5).Output from the sensor is Analog value they can be vary from 0 to 1023 . These 1024 values can be converted into binary format. Because, the arduino controller only support the machine level language. The binary value of 0 to 1023 can be consider as a output voltage varies from 0 to $5 \mathrm{~V}$. Digital Sensor's can't able to interface Analog pin's (A0, A1, A2, A3, A4 and A5). They are only read the analog sensor value. Otherwise the digital sensors are interface to the digital pin's.

\section{Sensor Interface}

In our process, we are using the three different sensor Temperature sensor, Humidity sensor and gas sensor. All the Sensors have a three different Pin outs. They are named as a VCC, GND, VOUT. VCC and GND is an supply power of the sensor. Where, VCC connected to the $5 \mathrm{~V}$ of arduino, GND connected to the ground. VOUT is an a output sensing value of the sensor. The ADC on the Arduino is a 10-bit ADC meaning it has the ability to detect $1,024\left(2^{\wedge} 10\right)$ discrete analog levels.

\section{IoT Monitoring}

The IoT monitoring is run based on the communication protocol operation. Arduino collect all the sensor value via analog port. They can be converted to digital signal and then they can be stored in arduino controller. This stored sensor values send to the IOT module through the operation of communication protocol. This way of connection is called TTL connection (Transistor- TransistorLogic).

\section{COMMUNICATION ALGORITHM}

PROTOCOL

In Arduino microcontroller, TX and RX (Pin number 0 and 1) act as a communication port. These pin's are send and receive the sensing data's to other devices. If you want to monitor the sensor value, now the TX pin of the arduino controller connected to the RX of the monitoring protocol device. If you want to control any object or load device, now TX pin of the controlling protocol device connected to the RX pin of the arduino.

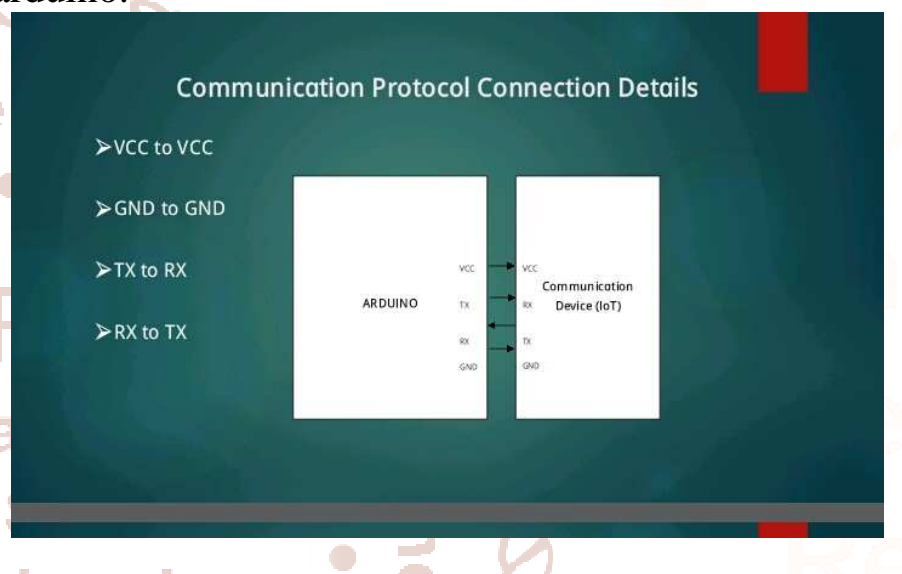

\section{WORKING EXPLANATION}

In this, we describe principle of working of our project. The Arduino board is programmed using Arduino coding. Gas (CO and NO2) sensors are developed and installed on AQMS circuit. The circuit is provided with a power supply. The values of sensors are sent to the mobile using the IOT central server. Then the values are displayed on the screen of the personal computer. When the Arduino controller initially activated and the all sensors will start to work according to the code transferred to the board. All sensors will start to collect the values depending on the parameters and it will update according to the delay values. There is particular range for particular sensors and it will act according to the threshold value. All the values sent to the mobile by using IOT central server. When there are any huge variations in the values it will update to the industrial management. The Arduino software (IDE) is easy-to-use for learners, yet lithe sufficient for innovative operators to take advantage of as well. For instructors, it's accessibly based on the Exemption program design situation, so scholar's knowledge to database in that location will be familiar with how the Arduino IDE works. 


\section{RESULT ANALYSIS}

This shows the final output scenario of the project. The working of the project is shown in this chapter Output scenario

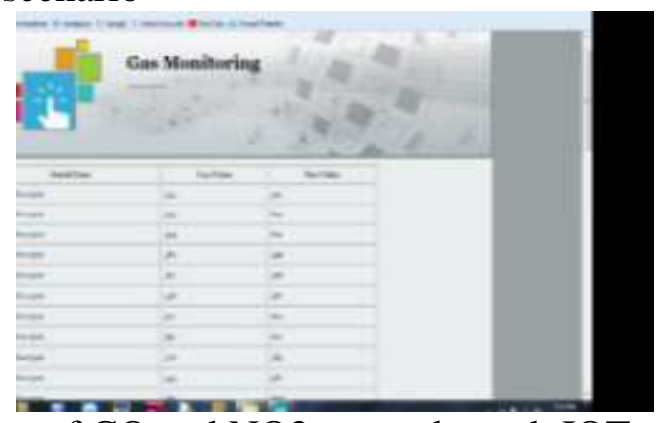

Values of CO and NO2 gases through IOT server

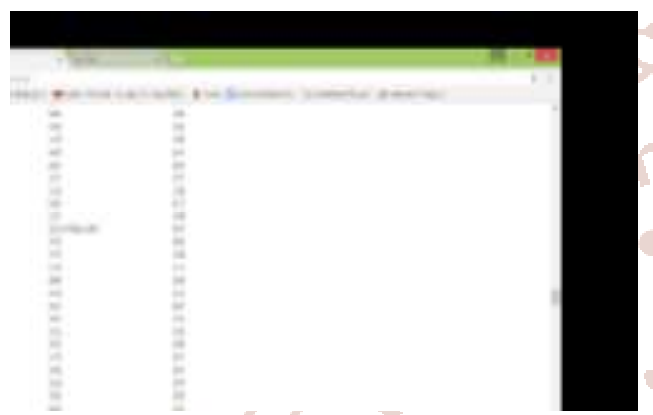

Values of $\mathrm{CO}$ and $\mathrm{NO} 2$ gases through IOT server in another place

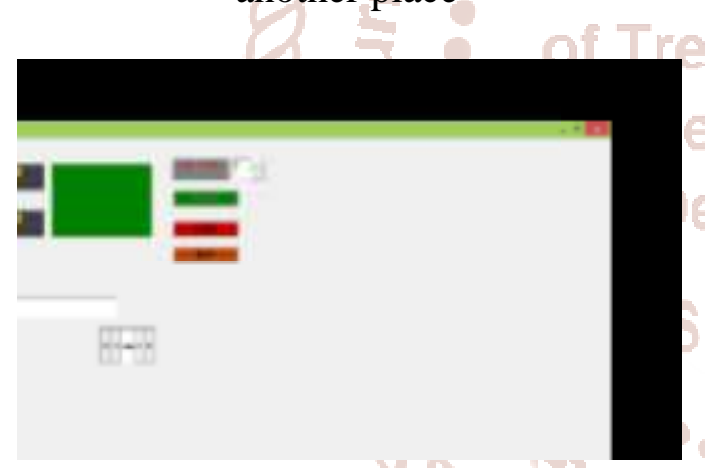

Values of $\mathrm{CO}$ and $\mathrm{NO} 2$ gases in personal computer without IOT

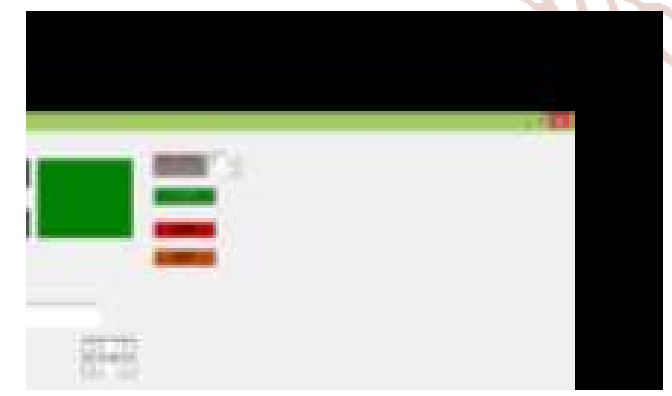

Values of $\mathrm{CO}$ and $\mathrm{NO} 2$ gases in personal computer without IOT

\section{CONCLUSION AND FUTURE WORK}

This paper introduces a Wireless Sensor Network (WSN)-based air quality monitoring system using IOT central server and gases sensors. This system is very simple as compared to the existing air quality monitoring systems. This project is also used for pollution monitoring purpose in cites. In future, this prototype can be extended in real time implementations of urban cities.

\section{REFERENCES}

1. Wen-TsaiSung, Jui-HoChen, Da- ChiunHuang, YiHaoJu,"Multisensors Real time Data Fusion Optimization for IOT Systems," 2014 IEEE International Conference on Systems, Man, and Cybernetics October 5-8, 2014, San Diego, CA, USA.

2. WeerasakCheunta, NitthitaChirdchoo and KanitthaSaelim, "Efficiency Improvement of an Integrated Giant Freshwater-White Prawn Farming in Thailand Using a Wireless Sensor Network," Research Gate, DECEMBER2014.

3. Jui-Ho Chen, Wen-Tsai Sung and Guo-Yan Lin, "Automated Monitoring System for the FishFarm Aquaculture Environment," 2015 IEEE International Conference on Systems, Man, and Cybernetics.

4. Mr. Kiran Patil,Mr. Sachin Patil,Mr. Sachin Patil and Mr. Vikas Patil,"Monitoring of Turbidity, $\mathrm{pH}$ \& Temperature of Water Based on GSM, "International Journal for Research in Emerging Science and Technology,Volume-2,Issue3,March-2015

5. Pradeep Kumar M, Monisha J, Pravenisha R, Praiselin V, Suganya Devi K, "The Real Time Monitoring of Water Quality in IoT Environment," International Journal of Innovative Research in Science, Engineering and Technology, Vol -5,Issue-6, March-2016.

6. Akanksha Purohit, UlhasKumar Gokhale,"Real Time Water Quality Measurement System based on GSM," IOSR Journal of Electronics and Communication Engineering, Vol. 9,Issue 3,PP 63-67,May-2014.

7. Sharudin Mohd Shahrul Zharif, "Intillegent Aquafarm System via SMS," Diss. Universiti Teknologi PETRONAS, 2007.

8. Sheetal Israni, Harshal Meharkure , Parag Yelore, "Application of IoT based System for Advance Agriculture in India, "International Journal of Innovative research in Computer and Communication Engineering Vol. 3, Issue. 11, November 2015. 